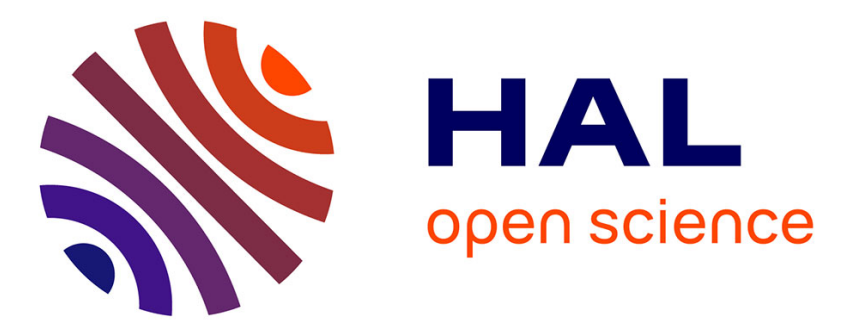

\title{
Temperature measurements of binary droplets using three-color laser-induced fluorescence
}

Christophe Maqua, Guillaume Castanet, Fabrice Lemoine, Nicolas Doué, Gérard Lavergne

\section{To cite this version:}

Christophe Maqua, Guillaume Castanet, Fabrice Lemoine, Nicolas Doué, Gérard Lavergne. Temperature measurements of binary droplets using three-color laser-induced fluorescence. Experiments in Fluids, 2006, 40 (5), pp.786 - 797. 10.1007/s00348-006-0116-y . hal-01570422

\section{HAL Id: hal-01570422 \\ https://hal.univ-lorraine.fr/hal-01570422}

Submitted on 29 Jul 2017

HAL is a multi-disciplinary open access archive for the deposit and dissemination of scientific research documents, whether they are published or not. The documents may come from teaching and research institutions in France or abroad, or from public or private research centers.
L'archive ouverte pluridisciplinaire HAL, est destinée au dépôt et à la diffusion de documents scientifiques de niveau recherche, publiés ou non, émanant des établissements d'enseignement et de recherche français ou étrangers, des laboratoires publics ou privés. 


\section{Temperature measurements of binary droplets using three-color laser-induced fluorescence}

\begin{abstract}
Evaporation of multicomponent droplets is a critical problem in many engineering applications, for example spray combustion. Knowledge of droplet temperature is a key issue in understanding the highly complex heat and mass-transfer phenomena related to multicomponent droplet evaporation and combustion. In this work, optical diagnosis based on three colorlaser-induced fluorescence was developed: the objective was to measure the temperature of binary droplets (ethanol and acetone mixtures), even when the composition varies with time. Demonstration on an overheated droplet stream of acetone-ethanol mixtures is described and the experimental data are compared with results from a numerical simulation based on the discretecomponents model.
\end{abstract}

\section{List of symbols}

C molecular concentration of the fluorescent tracer

$E=L / D$ non-dimensional distance

$D \quad$ droplet diameter

$f \quad$ temperature sensitivity function

$I_{0} \quad$ incident laser beam intensity

$I_{\mathrm{f}} \quad$ fluorescence intensity

$K_{\text {opt }} \quad$ optical constant

$K_{\text {spec }} \quad$ spectroscopic constant

$L \quad$ droplet spacing

$V_{\mathrm{c}} \quad$ collection volume

$R \quad$ fluorescence ratio

$t_{0} \quad$ initial time of the numerical simulation

C. Maqua $\cdot$ G. Castanet $\cdot$ F. Lemoine $(\varangle)$

LEMTA, CNRS UMR 7563, 2 Avenue de la Forêt de Haye, BP 160, 54504 Vandœuvre-les-Nancy Cedex, France

E-mail: fabrice.lemoine@ensem.inpl-nancy.fr

N. Doué · G. Lavergne

ONERA/DMAE, 2, avenue Edouard Belin, BP 4025, 31055

Toulouse cedex, France

\author{
$T \quad$ temperature \\ V velocity \\ Greek symbols \\ $\beta \quad$ temperature sensitivity coefficient \\ $\gamma \quad$ function taking into account the influence of the \\ ethanol volume fraction on the fluorescence \\ emission \\ $\lambda \quad$ wavelength \\ $\chi \quad$ ethanol volume fraction
}

\section{Introduction}

The problem of heat and mass transfer between a dispersed phase and a gas phase is encountered in numerous industrial processes, particularly in the field of spray combustion. In this field, understanding of the mechanism of transient evaporation of multicomponent droplets is fundamental, especially when the components have different volatilities. Droplet temperature is a critical property when trying to understand these highly complex phenomena, because the physical properties of the different components, for example diffusivities in the liquid phase, latent heat of vaporization, and saturated vapor pressure, depend on temperature. The rate of vaporization is also related to the mass fraction of each component at the droplet surface, which is also a function of droplet temperature (Sirignano 1999). 
Transient heating and evaporation phenomena in multicomponent droplets is very complex, and, therefore, must be investigated on basic devices such as monodisperse droplet streams (Virepinte et al. 2000; Silverman and Dunn-Rankin 1994; Mulholland et al. 1988), optically trapped droplets (Wilms et al. 2004) or acoustically levitated droplets (Kastner et al. 2000).

In monodisperse streams the droplets have the same diameter, temperature, and velocity at the injection point, and the effects of the different conditions can be easily separated. Physical models can be derived from such experimental investigations on simple geometrical configurations and for subsequent implementation in numerical simulation codes, which run in more complex configurations.

Experimental investigations require diagnosis, which are able to measure the droplet temperature. The most usual technique for determining the temperature of moving droplets is rainbow refractometry, based on the refractive index measurement by location of the rainbow pattern (Walker 1976; Van Beeck and Riethmuller 1995, 1997). Another method is based on exciplex fluorescence of pyrene added in the fuel ( $\mathrm{Lu}$ and Melton 2000). The major drawback of this technique, however, is that it becomes inefficient in sprays where oxygen is present, because of the strong quenching of the fluorescence by this compound, and extension to multicomponent fuels cannot be found in the literature.

Application of optical diagnosis to multicomponent droplets is rare and only light scattering-based techniques are reported in the literature. Zhao and Qiu (2004) used measurement of the droplet refractive index by rainbow refractometry to determine the temperature of binary mixtures of water and ethanol. Initial calibration of the refractive index as a function of temperature for each mixture is needed to infer droplet temperature. The authors were, however, unable to separate the effects of composition and temperature, which can be critical in the transient heating phase of the droplet. Wilms et al. (2004) also investigated rainbow refractometry to determine the composition of binary droplets of $n$-hexadecane and $n$-dodecane. Because the refractive index depends on both composition and temperature, droplet temperature was evaluated using pure $n$-hexadecane in the phase of constant-temperature evaporation, assuming the droplet temperature is similar for the other mixtures. Rainbow refractometry techniques are, furthermore, also highly sensitive to non-sphericity of the droplets and to the presence of refractive index gradients, especially in the transient heating phase. Any deviation from refractive index spatial homogeneity and from the sphericity can result in important bias in the measurements.

The development of two-color laser-induced fluorescence, initiated by Lavieille et al. (2001), enables measurement of single-component droplet mean temperature. This method uses fuel doped with a low concentration of a fluorescent organic dye, for example rhodamine $\mathrm{B}$, which is temperature-sensitive. The ratio of the signals collected on two spectral bands of detection, which have different temperature sensitivities, enables removal of the effect of unknown conditions and properties, for example laser intensity, fluorescent tracer concentration, and probe volume dimensions.

The goal is to extend the principles of the two-color fluorescence technique, i.e. one fluorescent tracer and two spectral bands of detection, to measurement of the temperature of binary droplets composed of ethanol and acetone. It is demonstrated that the fluorescence signal, like the rainbow signal, depends on both droplet composition and temperature. The objective of this paper is to show it is possible to separate the dependence on composition and temperature, by using a third spectral band for detection. The main principles and calibration phases of the technique are discussed in detail and a test experiment is described for an evaporating monodisperse binary droplet jet of ethanol and acetone mixtures, streaming linearly. Finally, the results are compared with those from a numerical simulation based on the discrete-components model.

\section{Principles of three color laser-induced fluorescence}

2.1 Application to single-component droplets: two-color laser-induced fluorescence

The liquid is seeded with a low concentration (a few $\mathrm{mg} \mathrm{L}^{-1}$ ) of rhodamine $\mathrm{B}$ before being disintegrated into droplets. Rhodamine B is an organic dye used as a fluorescent temperature sensor. Fluorescence of rhodamine B can, furthermore, easily be induced by use of the green line of the argon ion laser $(\lambda=514.5 \mathrm{~nm})$. The rhodamine B fluorescence spectrum is broadband. The fluorescence intensity, collected on a spectral band $\left[\lambda_{i 1} ; \lambda_{i 2}\right], i$ denoting the spectral band, is given by (Castanet et al. 2003):

$$
\begin{aligned}
I_{\mathrm{f} i} & =\int_{\lambda_{i 1}}^{\lambda_{i 2}} K_{\mathrm{opt}}(\lambda) K_{\mathrm{spec}}(\lambda) V_{\mathrm{c}} I_{0} C \mathrm{e}^{\beta(\lambda) / T} \mathrm{~d} \lambda \\
& \approx K_{\mathrm{opt}, i} K_{\mathrm{spec}, i} V_{\mathrm{c}} I_{0} C \mathrm{e}^{\beta_{i} / T}
\end{aligned}
$$

where $K_{\mathrm{opt}, i}$ is an optical constant taking into account the properties of the detection system (e.g. solid angle of detection and transmission of the optical components) and $K_{\mathrm{spec}, i}$ is a constant depending solely on the spectroscopic properties of the fluorescent tracer in its solvent, both for the spectral band $i . I_{0}$ is the laser excitation intensity, $C$ is the molecular concentration of the tracer, $T$ is the absolute temperature, and $V_{\mathrm{c}}$ is the volume from which the fluorescence photons are collected. This volume is the intersection of the laser beams, the droplet and the volume defined by the collecting optics. The product $C V_{\mathrm{c}}$ is related to the number of fluorescence photons collected by the photodetector. The $\beta(\lambda)$ factor characterizes the temperature dependence of the fluorescence intensity at the wavelength $\lambda$ (Lavieille et al. 2001) and $\beta_{i}$ characterizes the 
temperature dependence of the fluorescence emitted on the spectral band $i$.

To properly measure the temperature of a moving and potentially evaporating droplet, the effect of $C V_{\mathrm{c}}$ and $I_{0}$ must be removed. The collection volume $V_{\mathrm{c}}$ is constantly changing as the droplet crosses the probe volume. The distribution of the laser intensity within the droplet depends, furthermore, on the relative position of the droplet and also on the shape of the laser beam, which is affected by the refractive and focusing effects of the droplet surface. To eliminate these, the fluorescence intensity is detected on two spectral bands for which the temperature sensitivity is very different. The ratio of the fluorescence intensities collected on the two optimized spectral bands is given by:

$R_{12}=\frac{I_{\mathrm{f}_{1}}}{I_{\mathrm{f}_{2}}}=\frac{K_{\mathrm{opt}, 1}}{K_{\mathrm{opt}, 2}} \frac{K_{\mathrm{spec}, 1}}{K_{\mathrm{spec}, 2}} \mathrm{e}^{\frac{\beta_{1}-\beta_{2}}{T}}$,

This ratio is independent of probe volume. The effects of local laser intensity and tracer concentration are also eliminated. Use of a single reference measurement at a known temperature eliminates the optical and spectroscopic constants.

2.2 Application to binary droplets: three-color laserinduced fluorescence

In this study mixtures of acetone and ethanol will be considered and the ethanol volume fraction will be denoted $\chi$.

Preliminary spectroscopic studies have shown that the fluorescence spectrum of rhodamine B in a solvent depends on both temperature and solvent composition (Lavieille et al. 2001, 2004). The $\beta_{i}$ factor describing the effect of temperature on fluorescence intensity is also likely to depend on the solvent composition.

For binary fuels, a function $\gamma(\lambda, \chi)$, depending on the fluorescence emission wavelength $\lambda$, must be added, to take into account the effect of mixture composition on fluorescence intensity.

The new expression for the fluorescence intensity, integrated on a given spectral band $i$, is:

$$
\begin{aligned}
& \int_{\lambda_{i 1}}^{\lambda_{i 2}} K_{\mathrm{opt}}(\lambda) K_{\mathrm{spec}}(\lambda) V_{\mathrm{c}} I_{0} C \gamma(\lambda, \chi) \mathrm{e}^{\beta(\lambda, \chi)} \mathrm{d} \lambda \\
& \quad \approx K_{\mathrm{opt}, i} K_{\mathrm{spec}, i} V_{\mathrm{c}} I_{0} C \gamma_{i}(\chi) \mathrm{e}^{\beta_{i}(\chi)} \\
& \quad(i=1,2),
\end{aligned}
$$

where $\gamma_{i}(\chi)$ is a function taking into account the effect of composition on the fluorescence emission of spectral band $i$.

To properly determine the temperature of such binary droplets, an additional equation is necessary, because dependence on the ethanol volume fraction $\chi$ has been added. The signal should be detected on a third spectral band for which the sensitivity to temperature $T$ and ethanol volume fraction $\chi$ is different from that for the two others. The fluorescence intensity on the third spectral band can be expressed using Eq. 3, with $i=3$.

Using the two forms of Eq. 3, two fluorescence ratios can be calculated:

$$
R_{i j}=\frac{I_{\mathrm{f} i}}{I_{\mathrm{f} j}}=\frac{K_{\mathrm{opt}, i}}{K_{\mathrm{opt}, j}} \frac{K_{\mathrm{spec}, i}}{K_{\mathrm{spec}, j}} \frac{\gamma_{i}(\chi)}{\gamma_{j}(\chi)} \mathrm{e}^{\frac{\beta_{i j}(\chi)}{T}} \quad(\mathrm{i}, \mathrm{j})=\{(1,2),(2,3)\},
$$

where $\beta_{i j}$ denotes $\beta_{i}-\beta_{j}$. To optimize choice of the three spectral bands, a preliminary spectroscopic study was performed: fluorescence spectra were recorded for several ethanol volume fractions and for a range of liquid temperatures.

The function $\beta(\lambda, \chi)$ was determined by recording fluorescence spectra at two different liquid temperatures and for different constant ethanol volume fractions $\chi$ (Fig. 1). Temperature $T$ was then held constant and the ratio of two spectra recorded at two different $\chi$, using the same optical layout; this enabled determination of the function $\gamma(\lambda, \chi)$, because the function $\beta(\lambda, \chi)$ had previously been calculated. The function $\gamma(\lambda, \chi) / \gamma\left(\lambda, \chi_{0}\right)$, where $\chi_{0}$ was fixed at $60 \%$, is plotted in Fig. 1, for three different compositions $(\chi=20,40$, and $80 \%$, respectively). The spectral zone between 530 and $550 \mathrm{~nm}$ is poorly sensitive to temperature and highly sensitive to ethanol volume fraction. Beyond $570 \mathrm{~nm}$, the temperature sensitivity is quite high with a moderate peak value at approximately $580 \mathrm{~nm}$. As expected, significant dependence of this sensitivity on ethanol volume fraction is clearly observed. The three spectral bands are selected according to the rule that there are two unknown properties $(\chi$ and $T$ ) and two equations, which must be as independent as possible to achieve maximum accuracy. The ratio $R_{23}$ must, then, be very sensitive to temperature, but also sensitive to acetone volume fraction. The sensitivity of the ratio $R_{12}$ to temperature must be much lower, but it can also be sensitive to mixture composition. By means of a trade-off of between these criteria and the commercial availability of the optical filters, the following spectral bands were selected:

- Band 1: $(525,535 \mathrm{~nm})$

- Band 2: $(535,545 \mathrm{~nm})$

- Band 3: $(570,700 \mathrm{~nm})$

Selection of band 1 is delicate, because signal intensity is very low in this spectral region. Consequently, no reliable values of $\beta$ below $540 \mathrm{~nm}$ for $\chi=60 \%$ and $\chi=90 \%$ can be provided (Fig. 1). The temperature sensitivity on this band is very low, however, which is of interest for maximizing the temperature sensitivity of the ratio $R_{12}$. 
Fig. 1 Sensitivity of fluorescence emission to temperature and to ethanol volume fraction as a function of wavelength $\left(\chi_{0}\right.$ is $60 \%$ in this example)

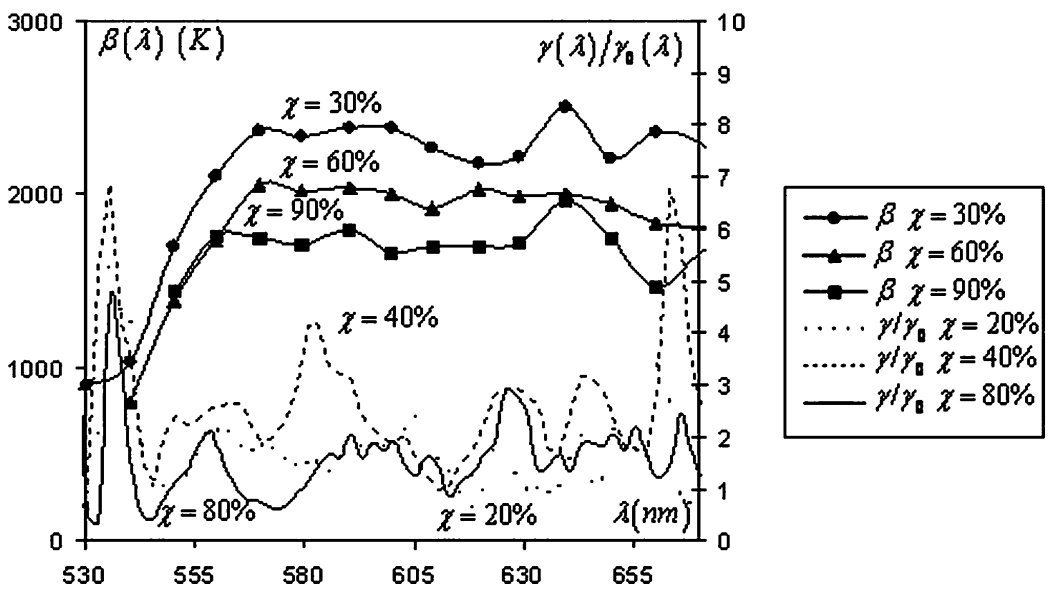

Furthermore, the total signal collected on band 1 is large enough to be exploited.

\section{Calibration process and optical set-up}

\subsection{Introduction}

All phases of the calibration process are performed in a quartz vessel. The laser excitation and reception optics are at a right angle to each other. Temperature is controlled by the use of a Peltier module (Fig. 2). The liquid temperature in the cell is monitored by means of a $\mathrm{K}$ type thermocouple, equipped with a transducer connected to a computerized data-acquisition board. All measurements are made with the probe volume placed as close to the detection windows as possible, to maintain optically thin conditions, i.e. to avoid re-absorption of the fluorescence, which could be different for the three spectral bands (Lavieille et al. 2001). The dye concentration during calibration is $10^{-6} \mathrm{~mol} \mathrm{~L}^{-1}$.

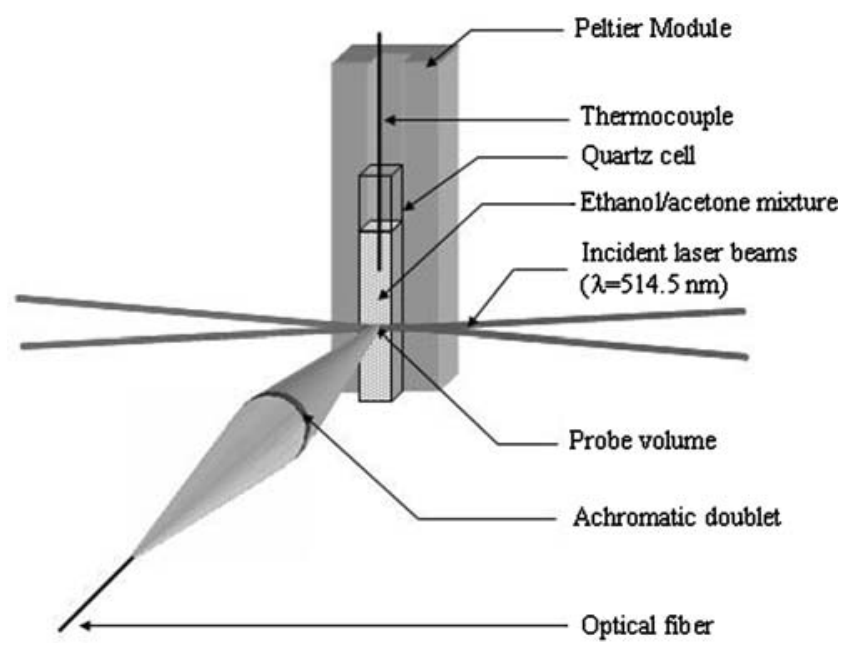

Fig. 2 Calibration cell
The calibration process is performed once only and is valid for a given set of spectral bands and acetone-ethanol mixtures.

\subsection{Optical set-up}

A laser Doppler anemometry (LDA) system was used to generate the probe volume, which enables measurement of droplet velocity concomitantly, with the use of additional detection optics and a Doppler signal processor. The probe volume is formed by the intersection of the laser beams and is $1,200 \mu \mathrm{m}$ long and $150 \mu \mathrm{m}$ in diameter (transverse direction). The fluorescence signal was detected by use of an achromatic doublet, located at right angle to the incident beams, connected to an optical fiber acting as a pinhole. The laser light $(\lambda=514.5 \mathrm{~nm})$ scattered by the droplets was high-pass filtered (cut-off at $\lambda=520 \mathrm{~nm}$ and full transmission beyond $525 \mathrm{~nm}$ ) to enable collection of the fluorescence emission only. The remaining fluorescence signal was separated into the three spectral bands by means of a set of neutral beamsplitters and interference filters (Fig. 3). Detection of the optical signal for the three selected spectral bands was performed by means of three cooled photomultipliers (Hamamatsu photosensors modules H9307-04) equipped with three pre-amplifiers (bandwidth $200 \mathrm{kHz}$ ). Acquisition, digitization, and sampling of the fluorescence signals were performed by means of a rapid computerized multi-channel acquisition board, at a sampling rate of $5 \mathrm{MHz}$.

\subsection{Determination of the function $\beta(\chi)$}

The functions $\beta_{12}(\chi)$ and $\beta_{23}(\chi)$ were determined by use of Eqs. 4. A reference temperature $T_{0}$ was defined and the two fluorescence ratios $R_{12}(T, \chi)$ and $R_{23}(T, \chi)$ were normalized by $R_{12}\left(T_{0}, \chi\right)$ and $R_{23}\left(T_{0}, \chi\right)$, respectively, which eliminated the system constants $K_{\text {opt }}$ and $K_{\text {spec. }}$ Finally, for a given ethanol volume 
Fig. 3 Block diagram of the three-color LIF experimental set-up

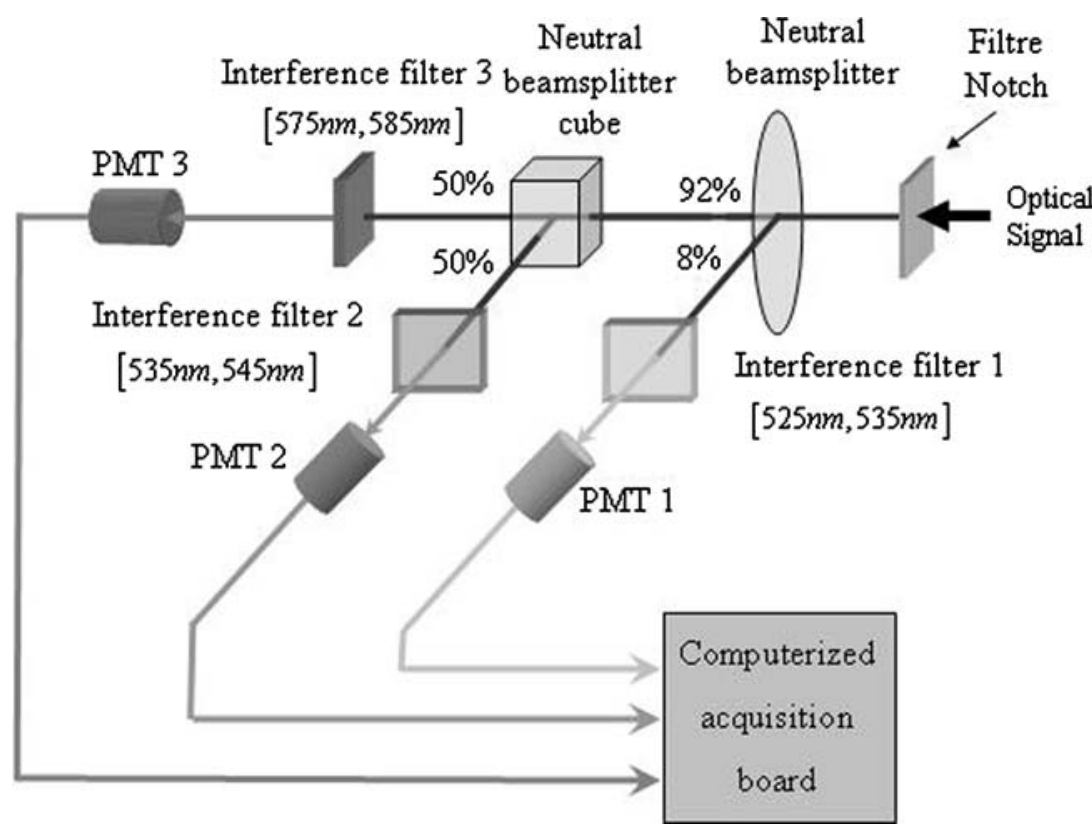

fraction $\chi$, the quantities $\ln \left(R_{i j}(T, \chi) / R_{i j}\left(T_{0}, \chi\right)\right)((i$, $j)=\{(1,2),(2,3)\})$ were plotted as a function of $(1 / T-1 /$

$T_{0}$ ) to determine the functions $\beta_{i j}(\chi)$ (Fig. 4). From Fig. 4 it is difficult to draw conclusions about the dependence of $\beta_{12}$ and $\beta_{23}$ on ethanol volume fraction $\chi$. The dependancies of $\beta_{12}$ and $\beta_{23}$ on $\chi$ have been specifically extracted from Fig. 4 and are represented in Fig. 5, where the dependence is clearly highlighted. The accuracy of this determination is limited by the slope calculation, which is unstable, but except for $\chi=60 \%$, the trend can be obtained with acceptable confidence. Polynomial fits are used to describe the behavior of $\beta_{12}(\chi)$ and $\beta_{23}(\chi)$
3.4 Determination of the function $\gamma(\chi)$

A reference ethanol volume fraction $\chi_{0}$ is given to normalize the fluorescence ratios $R_{12}(T, \chi)$ and $R_{23}(T, \chi)$ of Eq. 4 by use of $R_{12}\left(T, \chi_{0}\right)$ and $R_{23}\left(T, \chi_{0}\right)$, respectively. The temperature $T$ is measured directly by the thermocouple. Knowing the functions $\beta_{12}(\chi)$ and $\beta_{23}(\chi)$ from the previous experiments, the functions $f_{12}(\chi)$ and $f_{23}(\chi)$ can be established:

$f_{i j}(\chi)=\frac{\gamma_{i}(\chi)}{\gamma_{i}\left(\chi_{0}\right)} \frac{\gamma_{j}\left(\chi_{0}\right)}{\gamma_{j}(\chi)}=\frac{R_{i j}(T, \chi)}{R_{i j}\left(T, \chi_{0}\right)} \mathrm{e}^{-\frac{\beta_{i j}(\chi)-\beta_{i j}\left(\chi_{0}\right)}{T}}$
$(i, j=\{(1,2),(2,3)\})$,
Fig. 4 Calibration of the fluorescence ratios $\ln \left(R_{12}\right)$ and $\ln \left(R_{23}\right)$ as functions of temperature

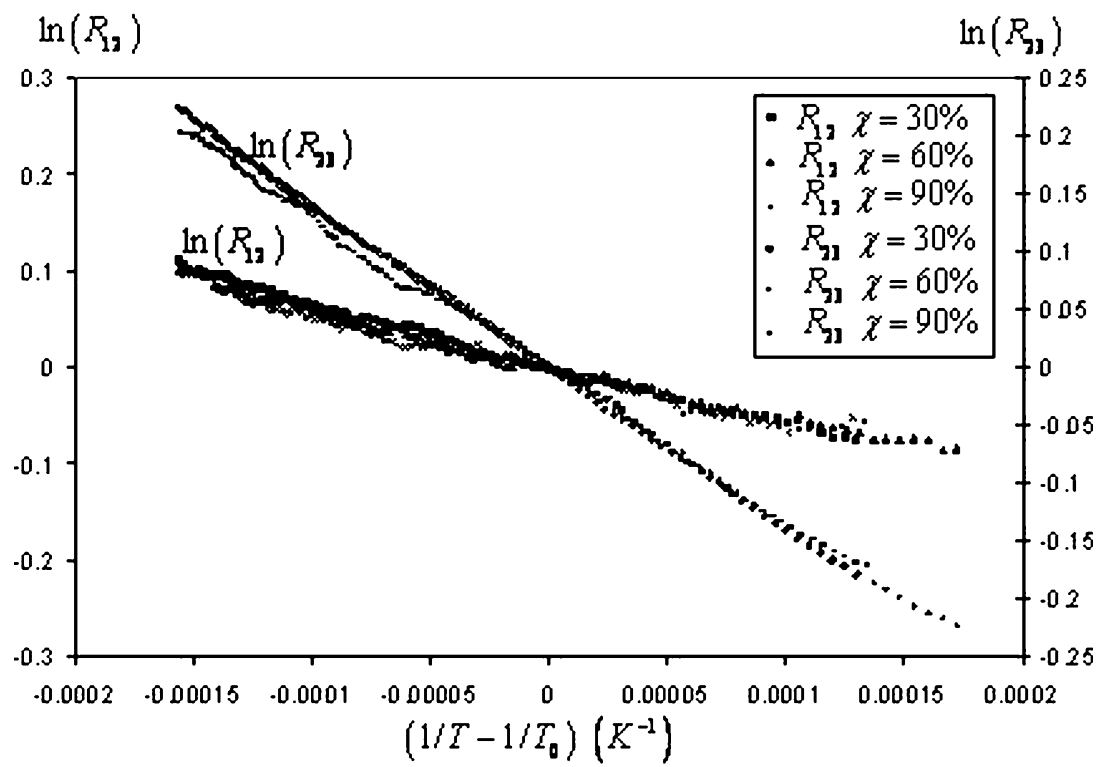


Fig. 5 Evolution of the temperature sensitivity of the two fluorescence ratios as a function of ethanol volume fraction in the mixture
Fig. 6 Dependence of the functions $f_{1}$ and $f_{2}$ on the ethanol volume fraction in the mixture
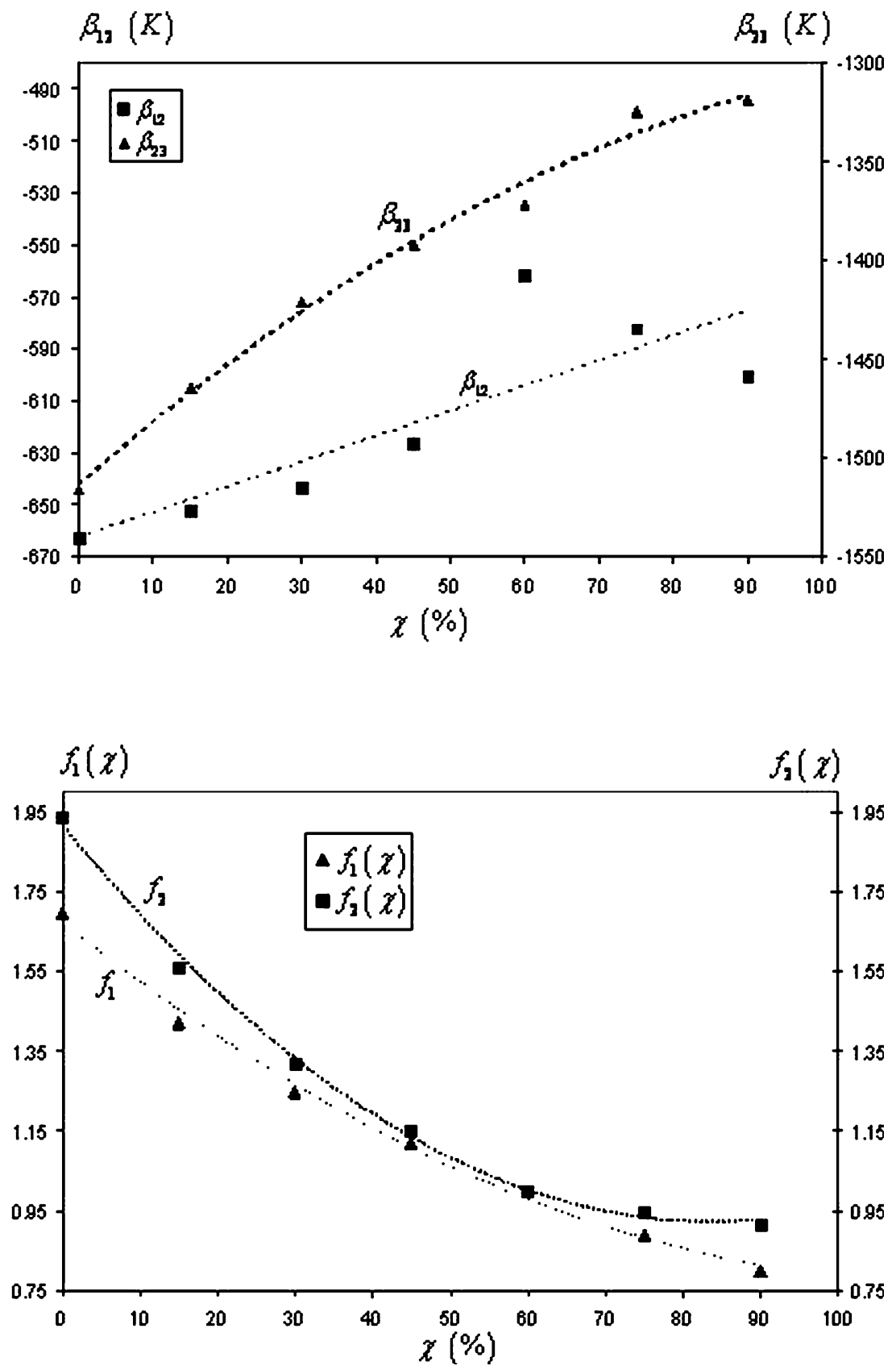

The functions $f_{12}(\chi)$ and $f_{23}(\chi)$ are plotted in Fig. 6; clear regular evolution of these functions is observable and the trend can also be fitted by polynomials.

\section{Measurement process and determination 7of the temperature}

The injection conditions are fixed, i.e. the injection temperature $T_{\text {inj }}$ and injection ethanol volume fraction $\chi_{\text {inj. }}$. To remove the system constants, i.e. $K_{\text {opt }}$ and $K_{\text {spec }}$, the measured fluorescence ratios $R_{12}$ and $R_{23}$ must be normalized by taking a reference measurement where the temperature and the mixture composition are known. This reference measurement must be taken at the closest possible position to the injection point. The best way is to take the reference measurement on the cylindrical liquid jet generated by the injector, before it disintegrates into droplets, where the ethanol volume fraction and temperature correspond to the injection conditions.

The normalized fluorescence ratios can be written as follows, by use of Eq. 4:

$\ln \left(\frac{R_{i j}}{R_{i j, \mathrm{inj}}}\right)=\ln \left(\frac{f_{i j}(\chi)}{f_{i j}\left(\chi_{\mathrm{inj}}\right)}\right)+\beta_{i j}(\chi)\left(\frac{1}{T}-\frac{1}{T_{\mathrm{inj}}}\right)$

$$
(i, j)=\{(1,2),(2,3)\} \text {, }
$$


where $R_{i j \text {,inj }}$ are the two fluorescence ratios measured at the injection point used for reference. Because the functions $f_{12}(\chi), f_{23}(\chi), \beta_{12}(\chi)$ and $\beta_{23}(\chi)$ have been intrinsically characterized by the initial calibration process, the two-equation system Eq. (6) can be solved by an iterative method and the two unknowns, i.e. the ethanol volume fraction $\chi$ and the property of interest, the droplet temperature $T$, can be inferred.

Droplet signal processing and averaging were performed as described elsewhere (Lavieille et al. 2001, 2002; Castanet et al. 2002).

\section{Discussion of error and uncertainties}

\subsection{Global uncertainties}

The global uncertainty of the method was tested in a temperature-controlled static cell, by measuring the fluorescence intensity distribution on the three spectral bands used for detection, for seven acetone-ethanol mixtures, each at seven different temperatures. The ethanol volume fraction $\chi$ ranged from 0 to $90 \%$ and the temperature $T$ ranged from 15 to $45^{\circ} \mathrm{C}$. From these 49 measurements at different temperatures and compositions, $49^{2}$ pairs of measurements can be formed randomly, one value being used for the reference and the other as a current measurement. Using the model described in the section Application to binary droplets ... and the calibrations reported in the section Calibration process and optical set-up, the temperature in the cell was calculated for the $49^{2}(2,401)$ combinations of reference and current measurements. The temperatures inferred from the measurements were compared with those given by the thermocouple and the standard deviation was calculated for the 2,400 readings. A similar scheme was applied to ethanol volume fraction. The standard deviation was $\sigma_{T}=1.3^{\circ} \mathrm{C}$ for the temperature and $\sigma_{\chi}=3.9 \%$ for the ethanol volume fraction. It is absolutely clear the proposed method is more sensitive to temperature variations than to the composition of the mixture; the value of the ethanol volume fraction must, therefore, be regarded as indicative only. The statistical dispersion of the temperature may be have different causes:

- Error in the measurement of reference temperature (thermocouple).

- Error in the composition of the solutions (moderate).

- Errors in the fluorescence intensity measurements by the PMTs, caused by non linearity and random noise.

- Errors in the determination of the functions $\beta$ and $f$.

The effect on the reference data (i.e. the quantities $T_{\text {inj }}$ and $\chi_{\text {inj }}$ should also be carefully studied. An error in $T_{\text {inj }}$, i.e. a possible shift in the temperature given by the thermocouple placed in the injector, will result in translation of all the temperature measurements by an amount approximately equal to the shift, if it does not exceed $4^{\circ} \mathrm{C}$. Therefore, the relative temperature variations remain valid if the error in the reference temperature remains below $4^{\circ} \mathrm{C}$.

\subsection{Laser energy distribution}

The effect of inhomogeneous distribution of laser energy within the droplet on the measured temperature must be also clearly examined. The laser energy internal field is affected by droplet diameter, laser beam diameter at the focus point, and liquid refractive index (Gouesbet et al. 1988; Lavieille et al. 2000; Castanet et al. 2005). If the droplet is not at equilibrium, i.e. if composition and temperature gradients are present within the droplet, inhomogeneous distribution of laser energy could affect the measured temperature, because the measured fluorescence is the result of a local product of laser energy and temperature and composition-dependent terms, according to:

$I_{f i}=\int_{V_{\mathrm{c}}} K_{\mathrm{opt}, i} K_{\mathrm{spec}, i} I(x, y, z) C \gamma_{i}(\chi(x, y, z)) \mathrm{e}^{\frac{\beta_{i}(\chi(x, y, z))}{T(x, y, z)}} \mathrm{d} x \mathrm{~d} y \mathrm{~d} z$,

where $(x, y, z)$ are the spatial coordinates within the droplet.

The local distribution of the laser energy within the droplet can be calculated using the model based on geometrical optics developed by Lavieille et al. (2000) and validated by Generalized Lorentz Mie Theory (Gouesbet et al. 1988; Castanet et al. 2005). The effect of inhomogeneous temperature and concentration distribution combined with inhomogeneous laser energy distribution will be studied by numerical simulation of the internal temperature field, developed at the end of the section Experimental results.

\section{Numerical simulations}

The experimental data were compared with a numerical simulation of the evolution of droplet mean temperature. The numerical simulation is based on the usual simplification assumptions: spherically symmetric system, quasi-steady gas phase, and one-third rule for calculation of gas-phase properties. The composition of the liquid is represented by the discrete components model developed by Sirignano (1999), which considers each individual species. Transport of the species and heat within the liquid phase is described by the effective diffusion model (Abramzon and Sirignano 1989). In this model, the temperature and composition profiles are spherically-symmetric and correction factors for the diffusion coefficients are used to take into account internal advection motion.

In the examples studied, the droplet spacing $L$ is quite low and the vaporization rate should be noticeably 
affected by droplet-to-droplet interaction phenomena. The correction factor $\eta$, determined by Atthasit et al. (2005), was therefore applied to the Sherwood and Nusselt numbers to evaluate the actual vapor flow rate and convective heating:

$\frac{\mathrm{Nu}}{\mathrm{Nu}_{\text {iso }}}=\frac{\mathrm{Sh}}{\mathrm{Sh}_{\text {iso }}}=\eta(E)$,

where the correction factor $\eta(E)$ is given by:

$\eta=1-0.57\left(1-\frac{1-\mathrm{e}^{-0.13(E-6)}}{1+\mathrm{e}^{-0.13(E-6)}}\right), \quad$ for $2.5 \leq E \leq 16$,

where $E=L / D$ is the non-dimensional distance. In this study, $E$ ranges from about 3 to 8 , which corresponds to $\eta$ ranging from 0.32 to 0.5 .

In Eq. $8, \mathrm{Nu}_{\text {iso }}$ and $\mathrm{Sh}_{\text {iso }}$ are the Nusselt and Sherwood numbers introduced by Abramzon and Sirignano (1989), for an isolated, moving, evaporating droplet. To focus only on heat and mass-transfer phenomena, the measured droplet velocity at each measurement location was used in the computation. The calculation is started with temperature and composition uniformly distributed over the entire droplet volume.

\section{Experimental results}

The experimental results were mainly obtained on an overheated monodisperse droplet stream injected at room temperature and atmospheric pressure. Downstream distance from the injection point was easily converted into time by using the local droplet velocity measured by LDA. The effect of mixture composition, droplet diameter, and droplet spacing were mainly studied.

First, the raw experimental results are presented in Fig. 7 for pure acetone, an $\chi=25 \%$ mixture, and pure ethanol $\left(T_{\mathrm{inj}}=45^{\circ} \mathrm{C}, \quad V_{\mathrm{inj}} \approx 12.2 \mathrm{~m} \mathrm{~s}^{-1}, \quad D_{\mathrm{inj}} \approx 139 \mu \mathrm{m}\right.$, $E \approx 7.8$ ). The cooling of the droplet can be clearly observed for the three examples tested, the largest tem- perature decrease occurring in the first step, during the liquid jet break-up phase. The temperature of pure acetone decreases faster than that of pure ethanol, the behavior of the composition with $\chi=25 \%$ was intermediate. These observations are compatible with expectations, because the droplet temperature is mainly governed by vaporization phenomena and the value of the saturated vapor pressure of acetone is three times that of ethanol at $45^{\circ} \mathrm{C}$.

Because it is difficult to evaluate and model heat and mass transfer during the break-up phase, all the experimental results will be normalized as follows:

$T^{*}=\frac{T\left(t=t_{0}\right)-T(t)}{T\left(t=t_{0}\right)-T_{\mathrm{amb}}}$,

where $t$ is the current time corresponding to a downstream position $z$ and $t_{0}$ is an initial instant selected after the break-up phase, where numerical simulation is started.

A first extensive set of normalized data is presented in Fig. 8 for different ethanol volume fractions in parallel with pure ethanol and acetone, for quite similar aerothermal injection conditions. These conditions are slightly scattered, because it seemed difficult to control them accurately while obtaining monodisperse droplets with fuels having different atomization properties (surface tension, viscosity...). The injection conditions are $V_{\mathrm{inj}} \approx 12.2 \mathrm{~m} \mathrm{~s}^{-1}, T_{\mathrm{inj}}=45^{\circ} \mathrm{C}, D_{\mathrm{inj}} \approx 139 \mu \mathrm{m}, E \approx 7.8$, and $T_{\mathrm{amb}}=22^{\circ} \mathrm{C}$. However, these moderate differences should not affect further analysis. The decrease in temperature seems to be correlated with ethanol volume fraction, the temperatures of the mixtures containing less ethanol decrease more rapidly. The experimental data are, however, noticeably difference for $\chi=0 \%$ (pure acetone) and $\chi=25 \%$ mixtures. The data for $\chi=50 \%, \chi=75 \%$, and $\chi=100 \%$, although well separated from those for $\chi=0 \%$ and $\chi=25 \%$, seem to behave quite similarly. A more gradual temperature decrease is obtained with pure ethanol. The numerical simulation results follow the same trend, although they seem to overestimate the experimental data. It is also confirmed that the temperature behavior
Fig. 7 Droplet temperature evolution for different volume fractions (the ethanol volume fractions indicated are those at the injection point)

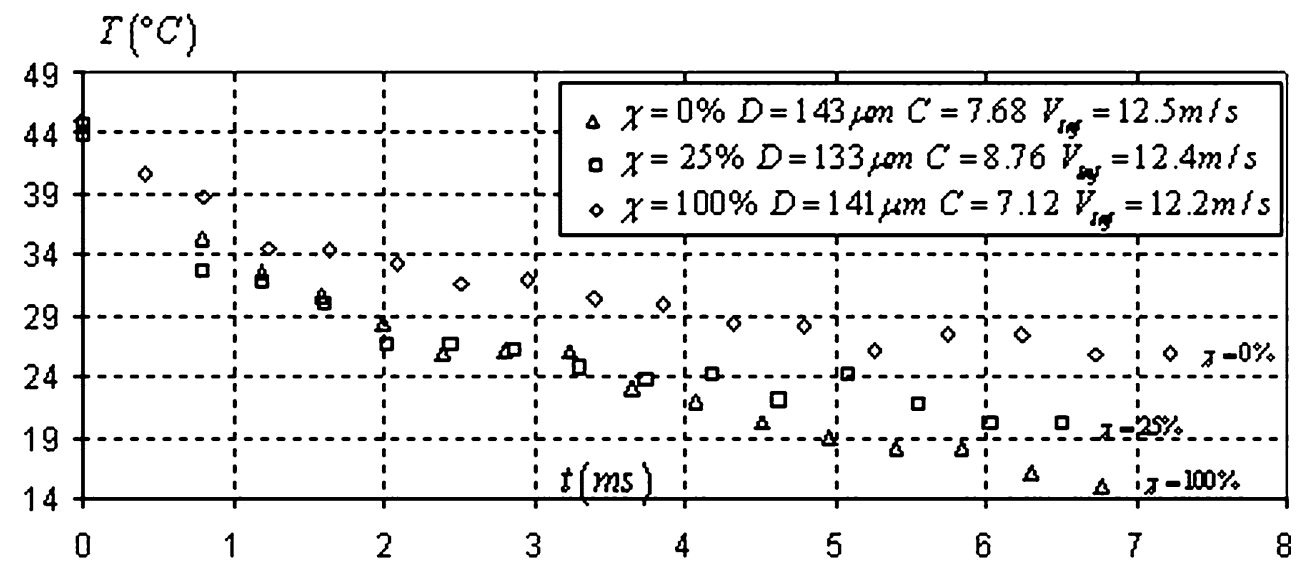


Fig. 8 Evolution of normalized droplet temperature for different ethanol volume fractions. Comparison with results from numerical simulation. (The ethanol volume fractions indicated are those at the injection point)

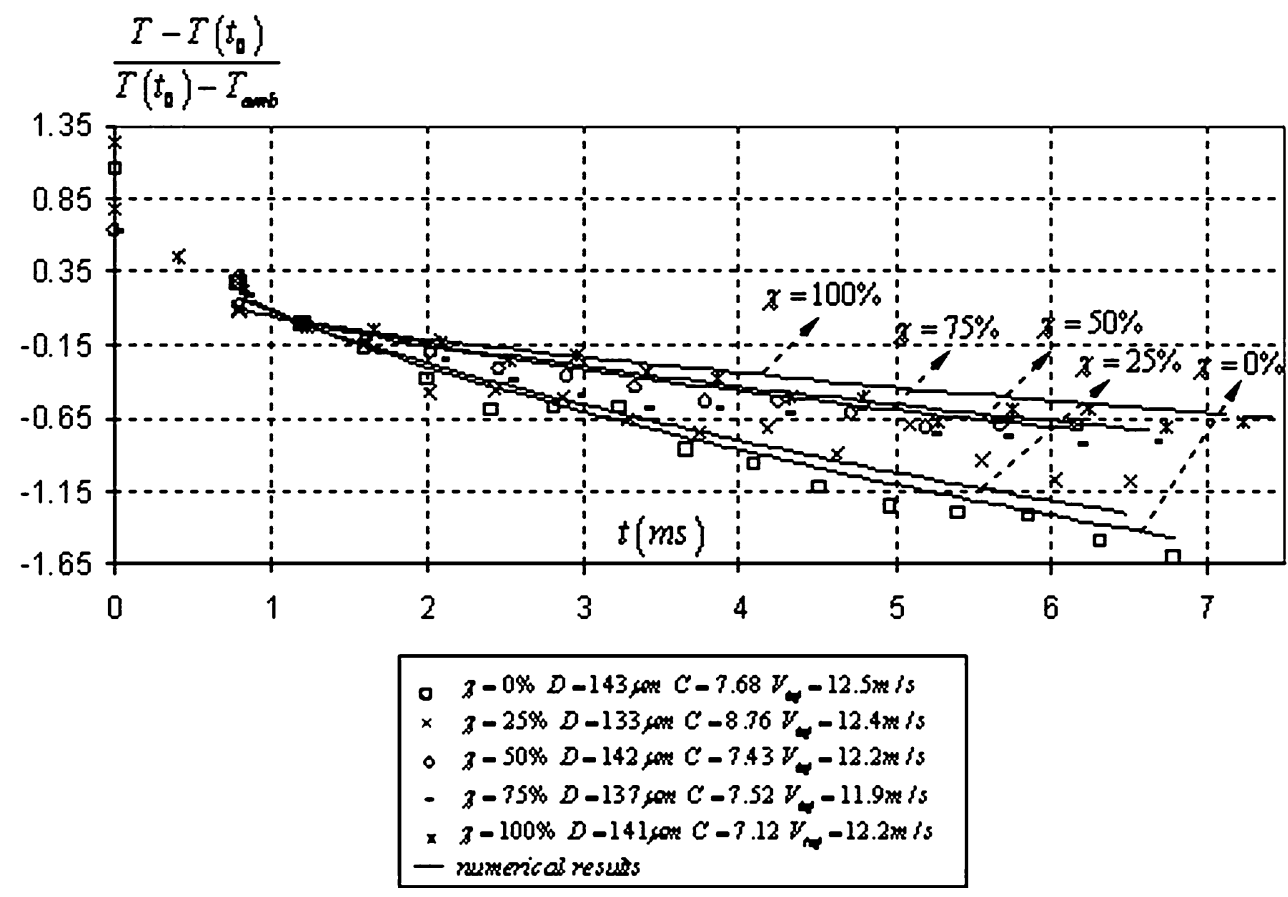

Fig. 9 Evolution of ethanol volume fraction for different compositions at injection. Comparison with numerical simulation results

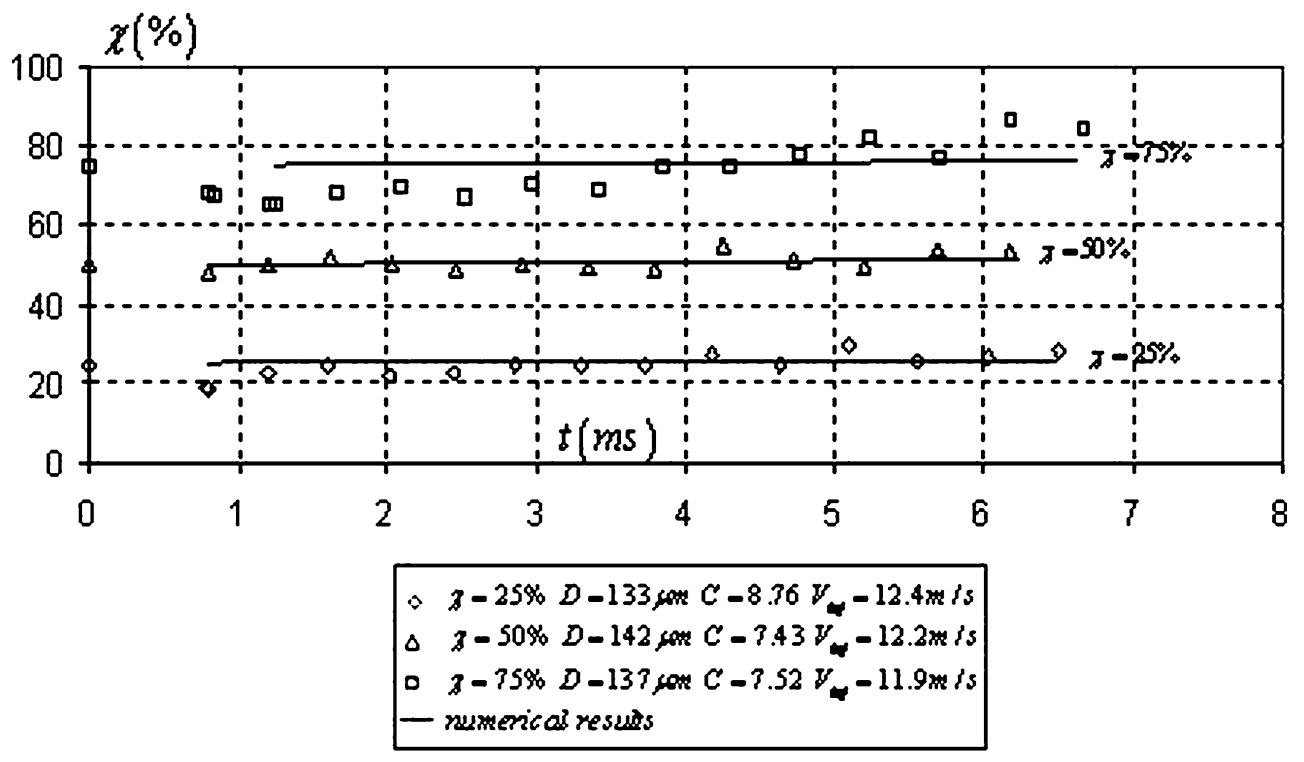

for $\chi=50 \%$ and $\chi=75 \%$ are very similar, which was already apparent from the experimental data. For the mixtures $\chi=25 \%, \chi=50 \%$, and $\chi=75 \%$, the ethanol fraction $\chi$ determined by solving the set of equations given by Eq. (6) is plotted in Fig. 9. During droplet evaporation, which is moderate, the ethanol volume fraction $\chi$ remains compatible with the ethanol volume fraction at injection, if the uncertainties in its determination are considered.

The effect of droplet diameter was examined for two different droplet sizes, one approximately $D_{\mathrm{inj}} \approx 90 \mu \mathrm{m}$ and the other approximately $D_{\mathrm{inj}} \approx 220 \mu \mathrm{m}$, both with a non-dimensional distance of approximately 3 (Fig. 10).
The $\chi=50 \%$ and $\chi=75 \%$ mixtures were considered. Conclusions similar to those of the previous study apply, for a given droplet diameter: an increase in ethanol fraction moderates the droplet temperature decrease. For the two mixtures investigated, the temperature decrease is steeper for the smallest droplets, which can easily be explained by the smaller volume-to-surface ratio, corresponding to the ratio between the droplet heat capacity and the droplet surface exchanges by evaporation and forced convection. The numerical results follow the same trend, even if agreement seems better for the bigger droplets. The difference between $\chi=50 \%$ and $\chi=75 \%$ lies in the range of uncertainty 
Fig. 10 Evolution of the normalized droplet temperature for different ethanol volume fraction and droplet diameter. Comparison with numerical simulation results. (The ethanol volume fractions indicated are those at the injection point)

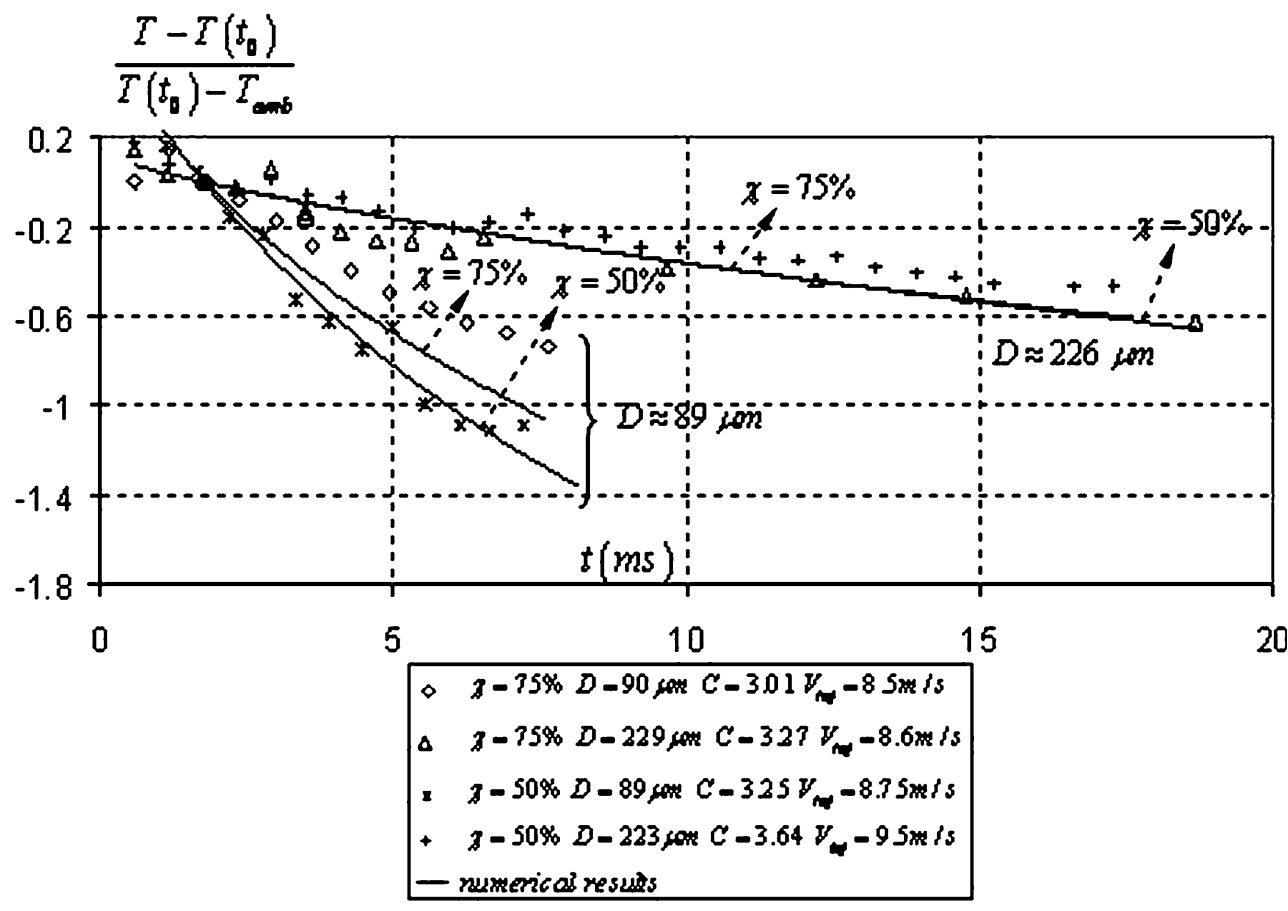

$\left( \pm 1.3^{\circ} \mathrm{C}\right)$. For the smaller droplets, the experimental data agree well with the calculated data for $\chi=50 \%$, but simulations agree well with experimental data but underestimate for $\chi=75 \%$.

The effect of droplet spacing was then investigated. It is known that increasing the non-dimensional distance tends to reduce the vaporization rate (Virepinte et al. 2000; Castanet et al. 2005). In the current experiment it was impossible to vary droplet diameter and droplet spacing independently. To overcome this difficulty, the time was normalized by the thermal diffusion time $\frac{D^{2}}{a_{\mathrm{eff}}}$, where $a_{\mathrm{eff}}$ is the thermal effective diffusivity, depending on the droplet Peclet number, defined by Abramzon and Sirignano (1989). Two nondimensional distances, $E \approx 4.5$ and $E \approx 8$, corresponding to two droplet diameters were investigated for two ethanol volume fractions, $\chi=25 \%$ and $\chi=75 \%$ (Fig. 11). For the two compositions, vaporization rate increases with droplet spacing. The numerical simulations are also in reasonable agreement with the experimental data, meaning that the correlation Eq. 9 correctly takes into account the increase of the
Fig. 11 Evolution of the normalized temperature, as a function dimensionless time, for different ethanol volume fractions and different distance non-dimensional distances. Comparison with the numerical simulation results. (The ethanol volume fractions indicated are those at the injection point)

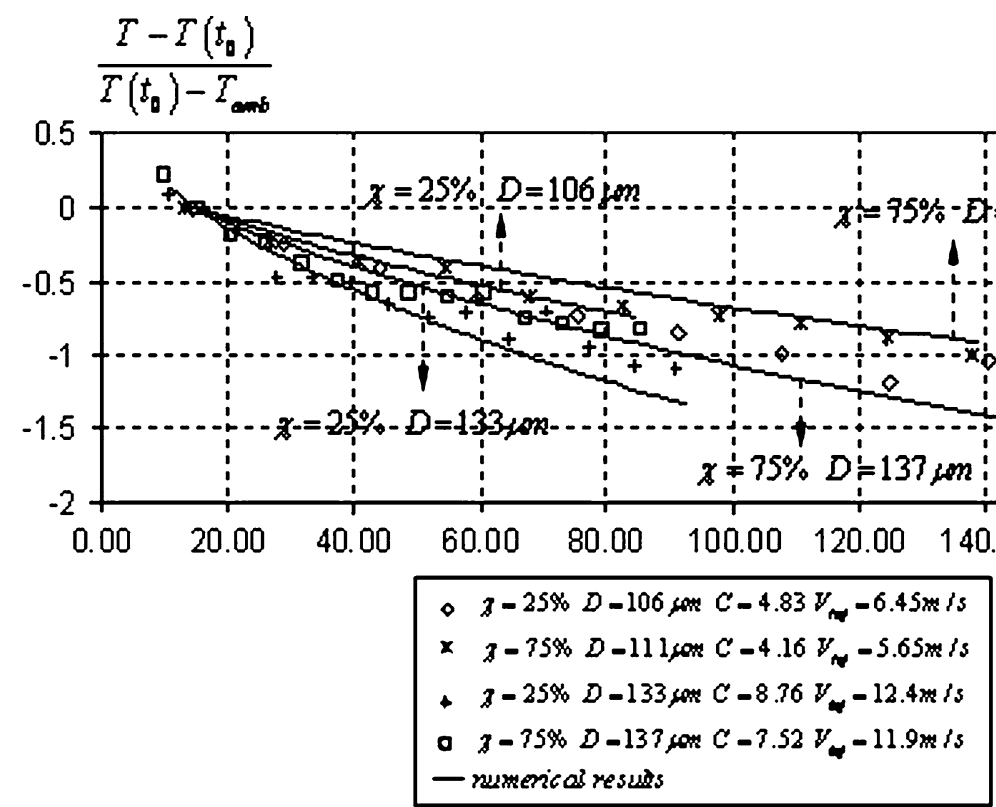


Fig. 12 Laser intensity distribution within the 89 and $223-\mu \mathrm{m}$ diameter droplets. The intersection of the laser beams is located in the droplet center (in the absence of the droplet)

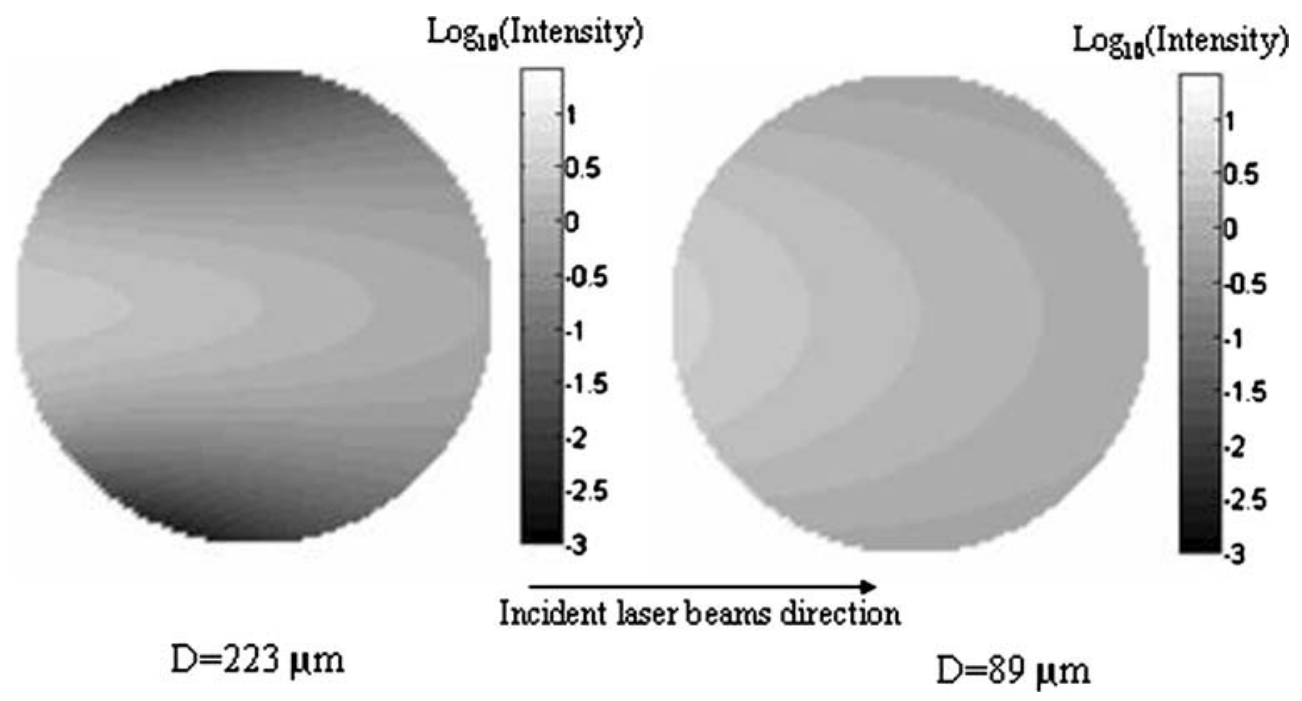

vaporization rate, which validates the correlation for binary droplets.

\section{Effect of inhomogeneous laser energy distribution}

The effect of inhomogeneous distribution of laser energy within the droplet was studied for two different droplet sizes $(D=89 \mu \mathrm{m}$ and $D=223 \mu \mathrm{m})$ for $\chi=50 \%$. An illustration of the spatial energy distribution for the two diameters is given in Fig. 12. As expected, illumination by the laser is more homogeneous for the smaller droplets. Transmission of fluorescence intensity at the liquidgas interface is not taken into account in this simulation, because most of the laser-illuminated points are in the collection angle. The local ethanol volume fraction $\chi$ $(x, y, z)$ and temperature $T(x, y, z)$ resulting from the numerical simulation have been used in Eq. 7 to evaluate the fluorescence intensity for both spectral emission bands and, subsequently, to determine the temperature that would be measured if spatial distributions of laser energy, composition, and temperature were taken into account. As apparent from Fig. 13 the numerical results taking into account the measurement system seem to overestimate the raw numerical results, the difference being more significant for the bigger droplet $D=223 \mu \mathrm{m}$. The experimental data corresponding to the two examples tested have also been plotted in Fig. 13, and it is clearly apparent that the deviations in the numerical results caused by the measurement system are within the accuracy of the technique. This could easily be explained by the moderate composition and temperature gradients in such an aerothermal situation; this point of view could be revised for a burning regime.
Fig. 13 Systematic error introduced by the measurement system to mean droplet temperature measurement

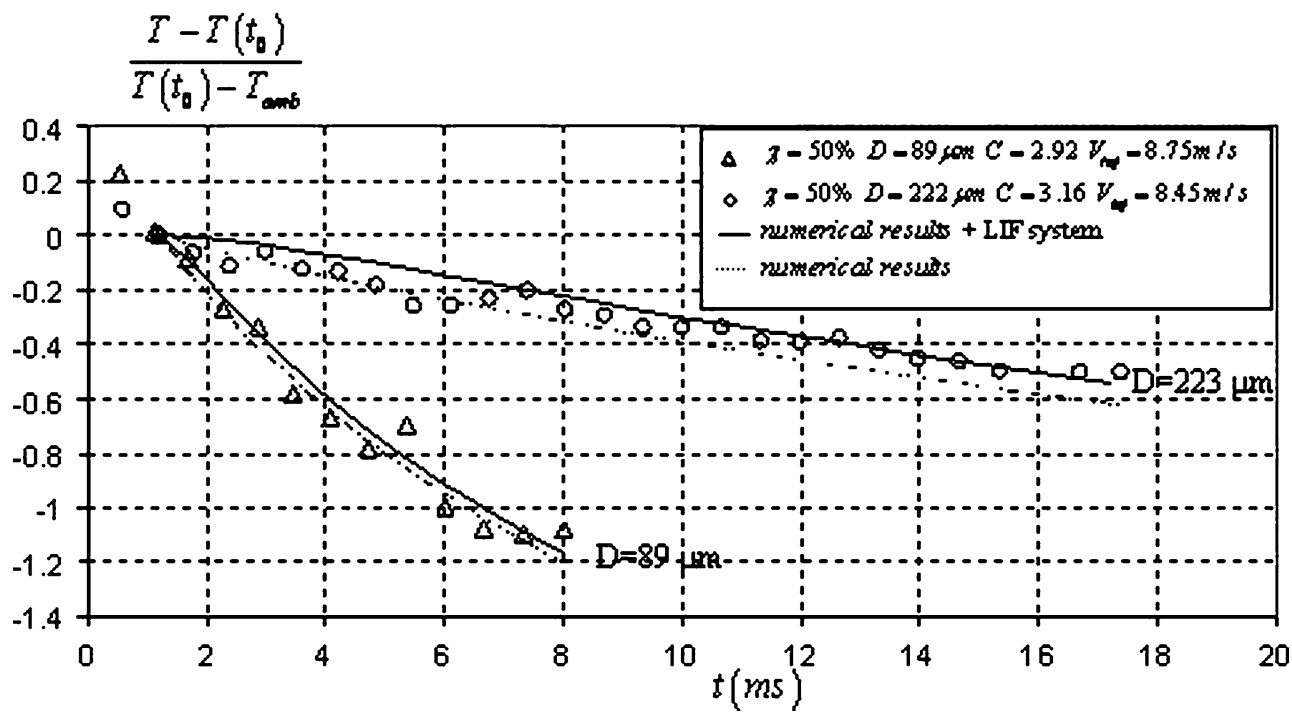




\section{Conclusions}

The transient temperature of moving evaporating binary droplets has been measured by a new laser-induced fluorescence technique, based on the detection of fluorescence emission of a tracer in three spectral bands. The effect of composition and temperature variations can be clearly separated and the temperature can be inferred with an accuracy of approximately $\pm 1.5^{\circ} \mathrm{C}$. Data inversion also gives an estimate of composition, which is currently only indicative. The investigated fuel was a mixture of two components of different volatility, acetone and ethanol. The technique has been tested on over-heated monodisperse droplets of an ethanol-acetone mixture, streaming linearly. Evaporative cooling is more intense when the concentration of the more volatile component is increased. For ethanol volume fractions above $50 \%$, few differences are observed in the temperature decrease. The effect of droplet diameter was consistent with our expectations, i.e. smaller droplets cool more quicker than the larger droplets for all the compositions tested. Comparison with the numerical simulations, based on the discrete components model, shows that the experiments and the numerical results follow a similar trend, although some discrepancies are apparent. These discrepancies may have different origins, some coming from the numerical model itself:

- heat and mass transfer within the droplets are assumed to be spherically symmetrical in the model, even though it is known that the internal velocity field is well described by Hill vortices (Chiang et al. 1992; Castanet et al. 2003);

- the calculation is initiated with uniform temperature and composition distribution, although in reality temperature and composition gradients are established during the break-up phase; and

- the physical properties of the mixture and their dependence on temperature may sometimes not be accurate, especially for binary diffusion coefficients.

Further research work will focus on extending this experiment to injection of the binary droplet stream in a hot air plume $(T \approx 750 \mathrm{~K})$ and on combining the temperature with measurements of size variation. Extension of the technique to other binary fuels will be also considered.

Acknowledgements This program has been conducted in the framework of the ASTRA program, supported by CNRS and ONERA.

\section{References}

Abramzon B, Sirignano WA (1989) Droplet vaporization model for spray combustion calculations. Int $\mathbf{J}$ Heat Mass Transfer 32:1605-1618
Atthasit A, Doué N, Biscos Y, Lavergne G (2005) Influence of droplet concentration on the dynamics and evaporation of a monodisperse stream of droplets in evaporation regime. Advances in combustion and atmospheric pollution, Semenov Memorial, Russia, Torus Press, Moscow, pp 19-36

Castanet G, Lavieille P, Lemoine F, Lebouché M, Atthasit A, Biscos Y, Lavergne G (2002) Energetic budget on an evaporating monodisperse droplet stream using combined optical methods. Evaluation of the convective heat transfer. Int $\mathbf{J}$ Heat Mass Transfer 45:5053-5067

Castanet G, Lavieille P, Lebouché M, Lemoine F (2003) Measurement of the temperature distribution within monodisperse combusting droplets in linear stream using two colors laserinduced fluorescence. Exp Fluids 35:563-571

Castanet G, Delconte A, Lemoine F, Mees L, Gréhan G (2005) Evaluation of temperature gradients within combusting droplets in linear stream using two colors laser-induced fluorescence. Exp Fluids 39:431-440

Chiang CH, Raju MS, Sirignano WA (1992) Numerical analysis of convecting, vaporizing fuel droplet with variable properties. Int J Heat Mass Transfer 35(5):1307-1324

Gouesbet G, Maheu B, Gréhan G (1988) Light scattering from a sphere arbitrarily located in a Gaussian beam, using a Bromwich formulation. J Opt Soc Am A5:1427-1443

Kastner O, Brenn G, Rensik D, Tropea C, Yarin AL (2000) Investigation of the drying behavior of suspension droplets in an acoustic levitator. In: 16th Annual conference on liquid atomization and sprays, Darmstadt

Lavieille P, Lemoine F, Lavergne G, Virepinte JF, Lebouché M (2000) Temperature measurements on droplets in monodisperse stream using laser-induced fluorescence. Exp Fluids 29:429-437

Lavieille P, Lemoine F, Lavergne G, Lebouché M (2001) Evaporating and combusting droplet temperature measurements using two-color laser-induced fluorescence. Exp Fluids 31(1):45-55

Lavieille P, Lemoine F, Lebouché M (2002) Experimental investigation on interacting low evaporating droplets temperature in linear stream using two colors laser induced fluorescence. Combust Sci Technol 174:117-142

Lavieille $\mathrm{P}$, Delconte A, Blondel D, Lebouché $\mathrm{M}$, Lemoine $\mathrm{F}$ (2004) Non-intrusive temperature measurements using threecolor laser-induced fluorescence. Exp Fluids 36:706-716

Lu QZ, Melton LA (2000) Measurement of transient temperature field within a falling droplet. AIAA J 38(1):95-101

Mulholland JA, Srivastava RK, Wendt JOL (1988) Influence of droplet spacing on drag coefficient in nonevaporating, monodisperse stream. AIAA J 26(10):1231-1237

Silverman MA, Dunn-Rankin D (1994) Experimental investigation of a rectilinear droplet stream flame. Comb Sci Tech 100:57-73

Sirignano WA (1999) Fluid dynamics and transport of droplets and sprays. Cambridge University Press, Cambridge

Van Beeck J, Riethmuller M (1995) Non-intrusive measurements of temperature and size of single falling raindrops. Appl Optics 34:1633-1639

Van Beeck J, Riethmuller M (1997) Rainbow interferometry with wire diffraction for simultaneous measurement of droplet temperature, size and velocity. Part Part Syst Charact 14:186-192

Virepinte JF, Biscos Y, Lavergne G, Magre P, Collin G (2000) A rectilinear droplet stream in combustion: droplet and gas phase properties. Comb Sci Tech 150:143-159

Walker JD (1976) Rainbow from single drops of water and other liquids. Am J Phys 44:421-433

Wilms J, Roth N, Arndt S, Weigand B (2004) Determination of the composition of multicomponent droplets by rainbow refractometry. In: 12th Symposium on application of laser techniques to fluid mechanics, Lisbon

Zhao Y, Qiu HH (2004) Measurements of multicomponent microdroplet evaporation by using novel optical techniques. In: 12th Symposium on application of laser techniques to fluid mechanics, Lisbon 